\title{
A substellar companion around the intermediate-mass giant star HD 11977
}

\author{
J. Setiawan ${ }^{1}$, J. Rodmann ${ }^{1}$, L. da Silva ${ }^{2}$, A. P. Hatzes $^{3}$, L. Pasquini ${ }^{4}$, O. von der Lühe ${ }^{5}$, J. R. de Medeiros ${ }^{6}$, \\ M. P. Döllinger ${ }^{4}$, and L. Girardi ${ }^{7}$ \\ 1 Max-Planck-Institut für Astronomie, Königstuhl 17, 69117 Heidelberg, Germany \\ e-mail: setiawan@mpia.de \\ 2 Observatório Nacional, R. Gal. José Cristino 77, 20921-400 São Cristóvão, Rio de Janeiro, Brazil \\ 3 Thüringer Landessternwarte, Sternwarte 5, 07778 Tautenburg, Germany \\ ${ }^{4}$ European Southern Observatory, Karl-Schwarzschild-Str. 2, 85748 Garching bei München, Germany \\ 5 Kiepenheuer-Institut für Sonnenphysik, Schöneck-Str. 6, 79104 Freiburg, Germany \\ ${ }^{6}$ Departamento de Física, Universidade Federal do Rio Grande do Norte, 59072-970 Natal, Brazil \\ 7 Osservatorio Astronomico di Trieste, via Tiepolo 11, 34131 Trieste, Italy
}

Received 2 February 2005 / Accepted 25 May 2005

\begin{abstract}
We report the discovery of a substellar companion to the intermediate-mass star HD 11977 (G5 III). Radial velocities of this star have been monitored for five years with FEROS at the 1.52-m ESO and later at the 2.2-m MPG/ESO telescope in La Silla, Chile. Based on the collected data we calculated an orbital solution with a period of $P=711$ days, a semi-amplitude of $K_{1}=105 \mathrm{~m} \mathrm{~s}^{-1}$, and an eccentricity of $e=0.4$. The period of the radial-velocity variation is longer than that of the estimated stellar rotation, rendering it unlikely that rotational modulation is the source of the variation in the radial velocity. This hypothesis is supported by the absence of a correlation between stellar activity indicators and radial-velocity variation. By determining a primary stellar mass of $M_{\star}=1.91 M_{\odot}$, the best-fit minimum mass of the companion and semi-major axis of the orbit are $m_{2} \sin i=6.54 M_{\mathrm{Jup}}$ and $a_{2}=1.93 \mathrm{AU}$, respectively. An upper limit for the mass of the companion of $m_{2} \lesssim 65.5 M_{\mathrm{Jup}}$ has been calculated from HIPPARCos astrometric measurements. Although the possibility of a brown-dwarf companion cannot be excluded, HD $11977 \mathrm{~B}$ is one of the few planet candidates detected around an intermediate-mass star. The progenitor mainsequence star of HD 11977 is probably an A-type star. This discovery gives an indirect evidence for planetary companions around early type main-sequence stars.
\end{abstract}

Key words. stars: general - stars: planetary system - stars: individual: HD 11977 - technique: radial velocity

\section{Introduction}

Among the substellar companions discovered around other stars, there are only a few planetary and brown-dwarf companions to intermediate-mass stars $\left(M_{\star} \approx 1.5-7 M_{\odot}\right)$. In fact, the presence of planetary or brown-dwarf companions to stars in this mass regime is not well explored in current radial-velocity planet search programmes. Ongoing radial-velocity surveys, e.g. CoRAliE \& HARPS survey and California \& Carnegie Planet Search, concentrate on solar-like stars. Only few planetary companions have been detected around evolved stars, like $\mathrm{G}$ and $\mathrm{K}$ giants (Hatzes et al. 2005, and references herein).

Intermediate-mass main-sequence stars have spectral classes from $\mathrm{A}$ to early $\mathrm{F}$ and higher effective temperatures than solar-type stars. Therefore, their spectra show fewer absorption lines than later, solar-like spectral types (late F, G and K). Due

^ Based on observations collected at the 1.52-m ESO telescope from October 1999 to February 2002 and at the 2.2-m MPG/ESO telescope from December 2003 to November 2004 at the La Silla Observatory, Chile. to faster stellar rotation $\left(v \sin i>30 \mathrm{~km} \mathrm{~s}^{-1}\right)$ their line profiles are also broader. These facts make it difficult to determine accurately the stellar radial velocity (RV).

Cool evolved stars, on the other hand, are suitable targets for precise RV measurements. As a star moves towards the red giant branch (RGB), its rotational velocity decreases and the stellar atmosphere cools significantly. Red giants, however, are chromospherically active (Pasquini et al. 2000). The variation of the chromospheric activity of G-K giants leaves an imprint in the spectral line profiles. Rotational modulation due to starspots can also produce RV variations, thereby mimicking the gravitational influence of low-mass companions. This effect can be investigated through the variations in the spectral line profile asymmetry and by monitoring the chromospheric activity indicators, like Ca II K and $\mathrm{H}$ emission lines, see e.g., Choi et al. (1995). Non-radial oscillations may also induce RV variations as shown by the asteroseismology of several giant stars (Hatzes \& Cochran 1994; Buzasi et al. 2000; Frandsen et al. 2002). 
In this letter we report the discovery of a substellar companion around HD 11977. We have excluded rotational modulation as the source of the RV variations, as described in the following sections.

\section{About the star HD 11977}

HD 11977 is a cool evolved star of spectral type G5 III (HIPPARCOS, ESA 1997). In the Hertzsprung-Russell diagram ( $M_{V}$ vs. $\left.T_{\text {eff }}\right)$ it is located in the "clump region" of the RGB. After ascending the RGB, the star moved down to the clump region where it is undergoing helium-core and hydrogen-shell burning. Clump giants typically spend $10^{8}$ years in this evolutionary stage, that, for initial masses higher than $1.7 M_{\odot}$, represents more than $5 \%$ of their main-sequence lifetime (Girardi et al. 2000).

The basic stellar parameters of HD 11977 are listed in Table 1. The spectral type, photometric data and parallax were taken from the HIPPARCos catalogues. Adelman (2001) reported a photometric variability of 0.5 millimag. Thus, HD 11977 is one of the least variable stars known from the HIPPARCos photometric survey.

The solar-metallicity evolutionary tracks by Girardi et al. (2000) show that the mass of HD 11977 is, indicatively, between 2.5-3.0 $M_{\odot}$. In a forthcoming paper (da Silva et al., in preparation) the $T_{\text {eff }}, R_{\star},[\mathrm{Fe} / \mathrm{H}]$ and $\log g$ are measured from the spectra of HD 11977. Their values are given in Table 1. Then, using as a constraint the observed $M_{V}, B-V,[\mathrm{Fe} / \mathrm{H}]$ values and their uncertainties, and the Girardi et al. theoretical isochrones, we derived the mass probability distribution function for HD 11977 in a more rigorous way. The subsolar metallicity of this $\operatorname{star}([\mathrm{Fe} / \mathrm{H}]=-0.21 \pm 0.1)$ lead us to derive a mass of $1.91 \pm 0.21 M_{\odot}$, and to exclude masses above $2.5 M_{\odot}$. A star of such mass and metallicity is not expected to have suffered significant mass loss during its ascent of the RGB. Therefore, the progenitor of HD 11977 was probably an A-type main sequence star.

The projected rotational velocity $v \sin i$ was computed using the cross-correlation method (e.g., Benz \& Mayor 1984; Setiawan et al. 2004). From the projected rotational velocity and the stellar radius we calculated the upper limit of the rotation period as $P_{\text {rot }} / \sin i=230-270$ days. Such a procedure of $P_{\text {rot }}$ determination is very sensitive on the $v \sin i$ precision. Nevertheless, the $v \sin i$ value we have obtained for HD 11977 is in excellent agreement with the value expected for a solartype G5III star with $B-V \sim 0.90, v \sin i=2.1 \mathrm{~km} \mathrm{~s}^{-1}$ (de Medeiros et al. 1996), pointing for an excellent precision on our $v \sin i$ determination.

\section{Observations and analysis}

We have monitored the radial velocity of HD 11977 from October 1999 to February 2002 with the echelle spectrograph FEROS $(R=\lambda / \Delta \lambda=48000)$ at the 1.52 -m ESO telescope. We continued the monitoring at the 2.2-m MPG/ESO telescope, to which FEROS had been moved, from December 2003 to November 2004.
Table 1. Stellar parameters of HD 11977.

\begin{tabular}{lll}
\hline \hline Spectral type & G5III & \\
$m_{V}$ & 4.68 & $\mathrm{mag}$ \\
$M_{V}$ & $0.57 \pm 0.07$ & $\mathrm{mag}$ \\
$B-V$ & $0.931 \pm 0.036$ & $\mathrm{mag}$ \\
Parallax & $15.04 \pm 0.47$ & $\mathrm{mas}$ \\
Distance & $66.5 \pm 2.1$ & $\mathrm{pc}$ \\
Effective temperature $T_{\text {eff }}$ & $4970 \pm 70$ & $\mathrm{~K}$ \\
Stellar radius $R_{\star}$ & $10.09 \pm 0.32$ & $R_{\odot}$ \\
Angular diameter & $1.49 \pm 0.02$ & $\mathrm{mas}$ \\
Metallicity $[$ Fe/H] & $-0.21 \pm 0.1$ & $\mathrm{dex}$ \\
Surface gravity $\log \left(g / \mathrm{cm} \mathrm{s}^{-2}\right)$ & $2.90 \pm 0.2$ & \\
Stellar mass $M_{\star}$ & $1.91 \pm 0.21$ & $M_{\odot}$ \\
log (Age/year) & $8.7-9.3$ & \\
$v_{\text {rot }}$ sin $i$ & $2.4 \pm 1.0$ & $\mathrm{~km} \mathrm{~s}^{-1}$ \\
Micro turbulence & $1.40-1.60$ & $\mathrm{~km} \mathrm{~s}^{-1}$ \\
$P_{\text {rot }} / \sin i$ & $230-270$ & days \\
\hline
\end{tabular}

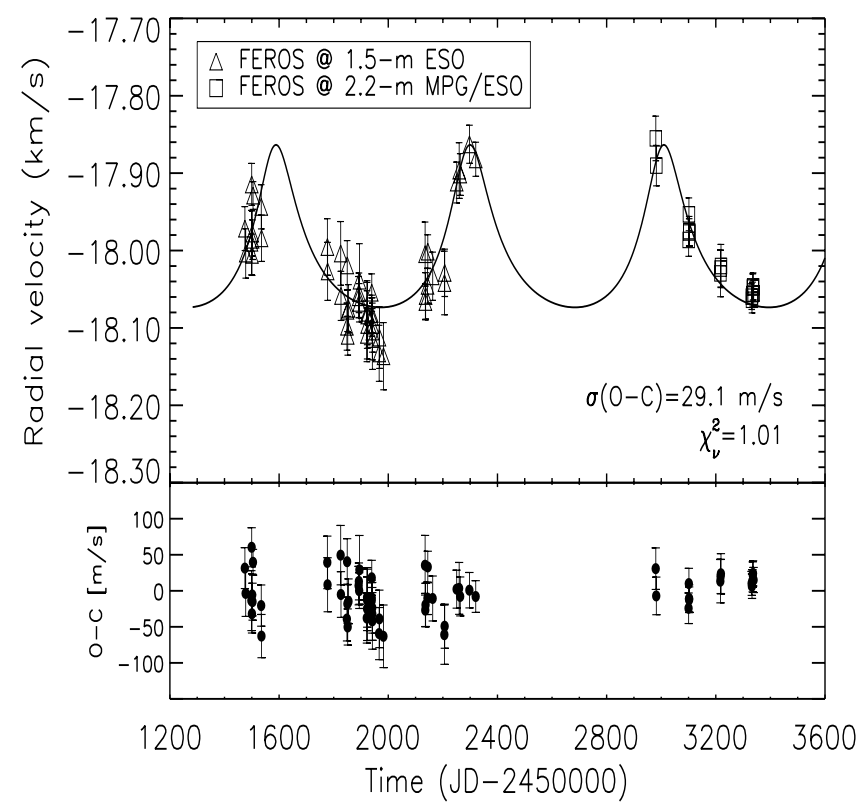

Fig. 1. Radial velocity measurements of HD 11977, taken with FEROS from October 1999 to November 2004. The measurements can be fitted with an orbital solution with a period of 711 days and an eccentricity of 0.4 . The lower panel shows the residual velocity. The rms scatter of the residuals is partly due to intrinsic variability on short time scales, as commonly found for giant stars.

FEROS is equipped with two fibres and can be operated in "object-sky" and "object-calibration" mode (Kaufer \& Pasquini 1998). We used the simultaneous calibration technique (Baranne et al. 1996) and the cross-correlation method to determine the radial velocity of HD 11977. Details of the data reduction and RV computation procedures have been described in Setiawan et al. (2003). The radial velocity measurements of HD 11977 are shown in Fig. 1. 


\section{Evidence for a substellar companion}

We observed a long-period variation in the radial velocity of HD 11977. A Lomb-Scargle periodogram (Scargle 1982) of the data yields significant power at a frequency of 0.0014 cycles day $^{-1}$ (Fig. 2) and a false alarm probability (FAP) of $\sim 10^{-9}$ using expressions given in Scargle (1982). This low FAP value was confirmed using the bootstrap randomisation technique (Kürster et al. 1997) and 200000 random shuffles of the data which yielded a FAP $<5 \times 10^{-6}$, i.e. no power in the random data greater than the data power.

It is unlikely that the RV variation of HD 11977 is due to rotational modulation. The upper limit of the rotation pe$\operatorname{riod}\left(P_{\text {rot }} / \sin i<300\right.$ days) is obviously much lower than the observed period in the RV variability. The analysis of the $\mathrm{Ca}$ II $\mathrm{K}$ emission lines (Fig. 3) did not show any significant stellar activity (e.g., due to surface inhomogeneities). We measured the relative flux K2V/K2R. This method has been applied on several red giants to detect rotational modulation (Setiawan et al. 2005). In addition, we measured the bisector velocity span (e.g. Queloz et al. 2001) and found no dependence between the asymmetry of the spectral line profile and the measured radial velocities (Fig. 4).

Assuming a semi-amplitude of $105 \mathrm{~m} \mathrm{~s}^{-1}$ and a rotational velocity of $2.4 \mathrm{~km} \mathrm{~s}^{-1}$, a simple starspot model results in a spotfilling factor of $4 \%$ of the stellar hemisphere (Hatzes 2002). For a starspot close to the equator and with $\Delta T=1200 \mathrm{~K}$, this translates to a photometric variation of $\sim 0.1$ mag (Launhardt $\&$ Setiawan, in preparation), which is of a factor of $\sim 200$ larger than the value given in Adelman (2001). However, we cannot exclude the possibility that the HIPPARCos photometric measurements were done at a time when HD 11977 was in the "quiet" phase of its activity cycle.

The astrometric variation of HD 11977 is less than 1 mas (HIPPARCOS). This gives a lower limit for $\sin i \gtrsim 0.1$. If the $\sin i$ were 0.1 , the true rotation velocity would be about $24 \mathrm{~km} \mathrm{~s}^{-1}$. A $\mathrm{K}$ giant rotating this fast would be very active. In this case, strong Ca II K emission lines should be expected. We did not observe this feature in our spectroscopic measurements.

We therefore exclude rotational modulation due to surface inhomogeneities as the source of the RV variation, and infer the presence of a substellar companion (planet or brown dwarf) orbiting HD 11977. The orbital solution to these data yielded a period of $P=711$ days, a semi-amplitude of $K_{1}=105 \mathrm{~m} \mathrm{~s}^{-1}$ and an eccentricity of $e=0.4$. The residual of $29.1 \mathrm{~m} \mathrm{~s}^{-1}$ is comparable with the expected long-term accuracy of FEROS. The computed orbital parameters yield a minimum mass of the companion $m_{2} \sin i=6.54 M_{\text {Jup }}$ (Table 2).

The HIPPARCos astrometry data yields an upper limit for the companion mass of less than $66 M_{\text {Jup }}$, i.e. below or near the hydrogen-burning limit. The expectation value of $\sin i=\pi / 4$ for randomly oriented orbital planes can be used to derive a value of $m_{2}$. We derived a value of $m_{2} \approx 8.3 M_{\text {Jup }}$. Since this value is below the deuterium-burning limit ( 13 $\left.M_{\text {Jup }}\right)$, HD 11977 B is one of very few planetary companions discovered around intermediate-mass giant stars.

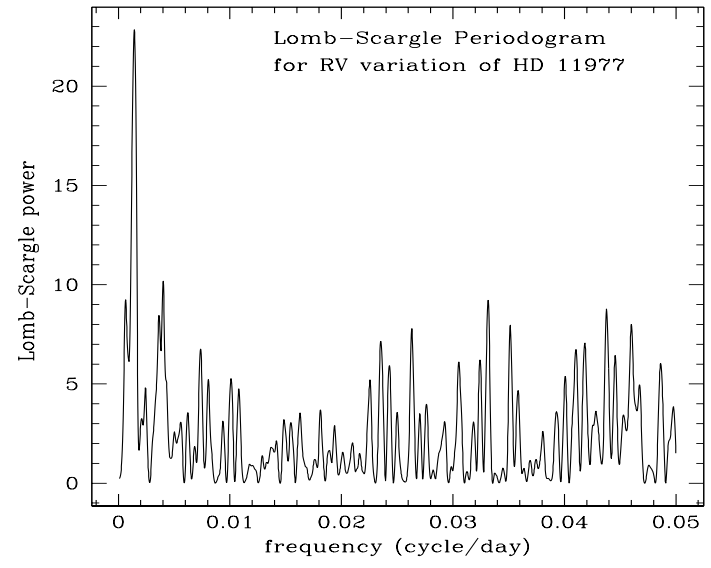

Fig. 2. The Lomb-Scargle periodogram of the radial velocity measurements. The periodogram shows a significant power at a frequency of 0.0014 cycles day $^{-1}$.
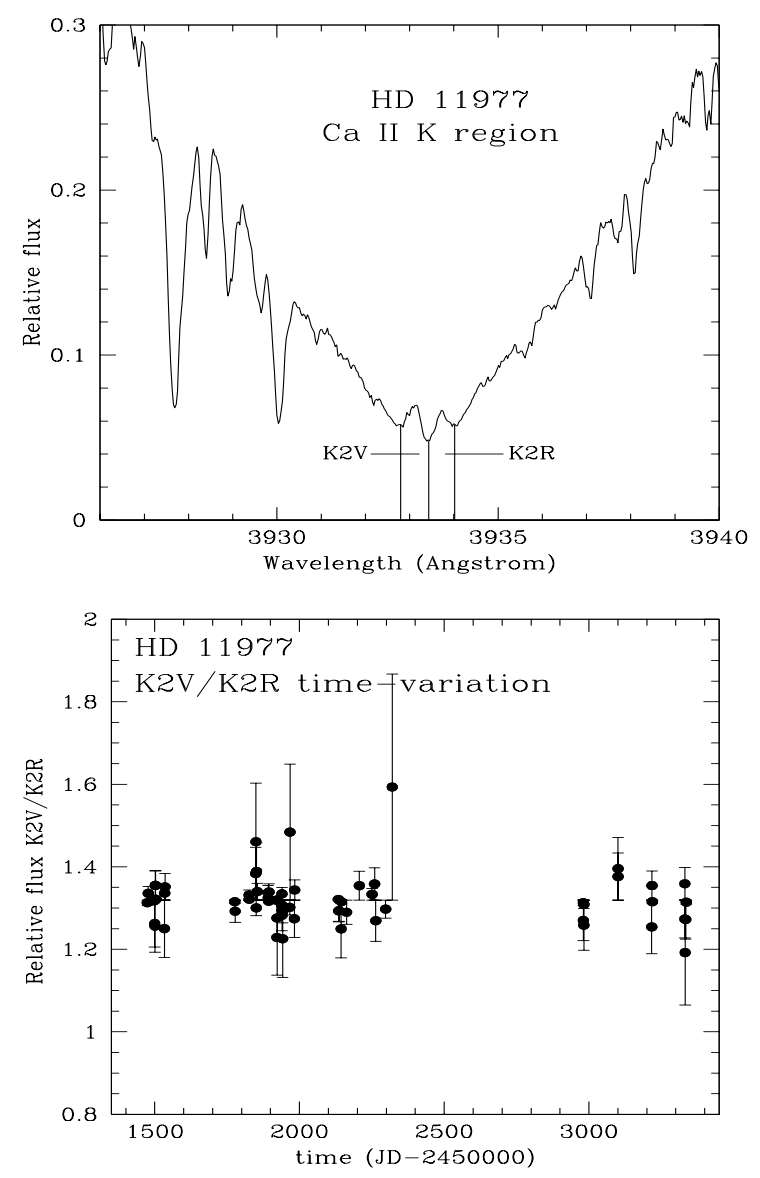

Fig. 3. The upper panel shows the region around the Ca II K emission lines. The relative flux K2V/K2R (lower panel) shows no long-period variation.

\section{Discussion}

To date there are only two substellar companions detected around intermediate-mass stars, namely HR 7329 (Lowrance et al. 2000) and HD 13189 (Hatzes et al. 2005). HR $7329 \mathrm{~A}$ is a main-sequence star of spectral type A0 of mass $M_{\star}=2.9 M_{\odot}$. HR $7329 \mathrm{~B}$ is a brown-dwarf companion with $m_{2} \approx 30-50 M_{\text {Jup }}$ 


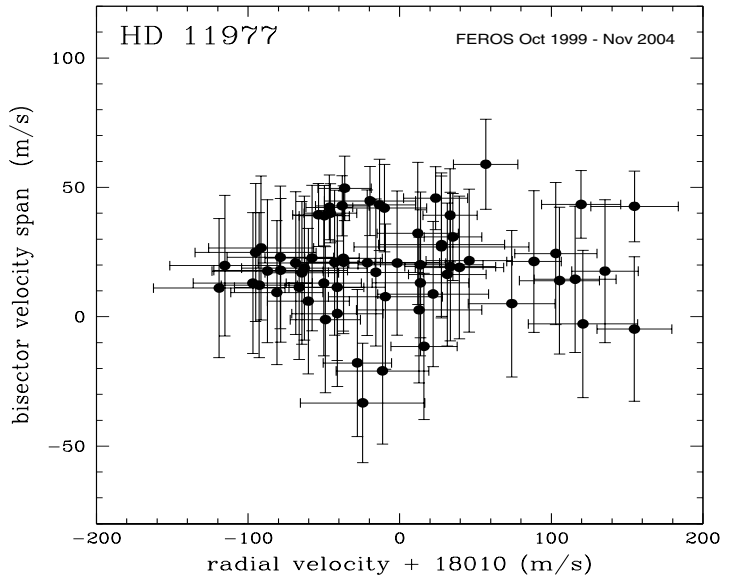

Fig. 4. Bisector velocity span (BVS) of HD 11977 calculated from the spectra taken between October 1999 and November 2004. The diagram shows no correlation between BVS and the measured radial velocities.

Table 2. Orbital parameters for HD 11977 B.

\begin{tabular}{lll}
\hline \hline$P$ & $711 \pm 8$ & days \\
$T_{0}(\mathrm{JD}-2450000)$ & 1420 & days \\
$e$ & $0.4 \pm 0.07$ & \\
$V$ & -18.010 & $\mathrm{~km} \mathrm{~s}^{-1}$ \\
$\omega_{1}$ & $351.5 \pm 9.5$ & $\mathrm{deg}$ \\
$K_{1}$ & $0.105 \pm 0.008$ & $\mathrm{~km} \mathrm{~s}^{-1}$ \\
$\sigma(\mathrm{O}-\mathrm{C})$ & 29.1 & $\mathrm{~m} \mathrm{~s}^{-1}$ \\
$f(m)$ & $(6.55 \pm 1.63) \times 10^{-8}$ & $M_{\odot}$ \\
$a_{1} \sin i$ & $9.41 \times 10^{5}$ & $\mathrm{~km}$ \\
\hline with $m_{1}=M_{\star}=1.91 M_{\odot}$ & & \\
$m_{2} \sin i$ & 6.54 & $M_{\text {Jup }}$ \\
$a_{2}$ & 1.93 & $\mathrm{AU}$ \\
\hline
\end{tabular}

in an orbit of $\sim 200 \mathrm{AU}$ (Guenther et al. 2001). HD $13189 \mathrm{~A}$ is a K giant/supergiant of mass $M_{\star}=2-7 M_{\odot}$. The distance to this star is only poorly known, thus its stellar mass cannot be determined accurately. This leads to large uncertainty for the mass of the substellar companion. The companion's minimum mass was estimated between 8-20 $M_{\text {Jup }}$. Due to this uncertainty HD 13189 B can be either a planet or brown dwarf.

HD 11977 is the first intermediate-mass star with accurate mass around which a planetary companion has been discovered (Fig. 5). Assuming negligible mass loss during the RGB phase, the main-sequence progenitor of HD 11977 is probably an A star with an stellar mass of approximately $2 M_{\odot}$ (Schmidt-Kaler 1982). HD 11977 has subsolar metallicity $([\mathrm{Fe} / \mathrm{H}]=-0.21)$. Low metal abundance has been also found in other giants hosting planets (Sato et al. 2003; da Silva et al., in preparation). This point will be investigated in more detail in our next study of stellar abundances.

Our discovery gives a crucial support to search for extrasolar planets around early type main-sequence stars and stars in late evolutionary stages. Detections of planetary companions to stars outside the solar-mass regime can provide valuable constraints for theories of planet formation.

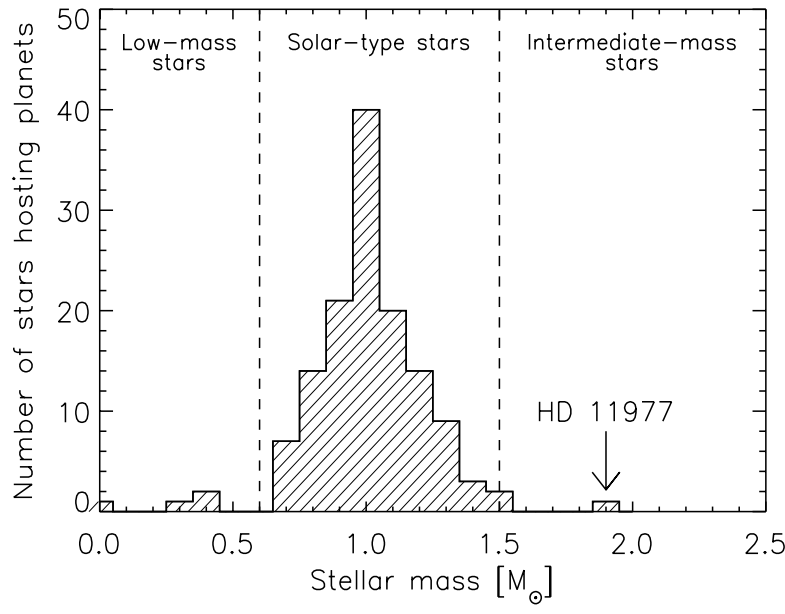

Fig. 5. Mass distribution of stars with known planetary companions (as of April 2005, including HD 11977). We exclude HD 13189 from this histogram due to the large uncertainty of the stellar mass.

Acknowledgements. We thank John Pritchard and Fernando Selman for their works on FEROS. For the long-period assistance during the observations with the 1.52-m ESO we thank Rolando Vega and Arturo Torrejon. We also thank Francisco Labrana, Mauro Stefanon, Karla Aubel and Manuel Pizzaro for the supports during the observations at the 2.2-m MPG/ESO.

\section{References}

Adelman, S. J. 2001, A\&A, 367, 297

Baranne, A., Queloz, D., Mayor, M., et al. 1996, A\&AS, 119, 373

Benz, W., \& Mayor, M. 1984, A\&A, 138, 183

Buzasi, D., Catanzarite, J., Laher, R., et al. 2000, ApJ, 532, 133

Choi, H.-J., Soon, W., Donahue, R. A., et al. 1995, PASP, 107, 744

de Medeiros, J. R., da Rocha, C., \& Mayor, M. 1996, A\&A, 314, 499

ESA, The Hipparcos Catalogue 1997, ESA SP-1200

Frandsen, S., Carrier, F., Aerts, C., et al. 2002, A\&A, 394, 5

Girardi, L., Bressan, A., Bertelli, G., et al. 2000, A\&AS, 141, 371

Guenther, E. W., Neuhäuser, R., Huélamo, N., et al. 2001, A\&A, 365, 514

Hatzes, A. P., \& Cochran, W. D. 1994, ApJ, 422, 366

Hatzes, A. P. 2002, AN, 323, 392

Hatzes, A. P., Guenther, E. W., Endl, M., et al. 2005, A\&A, 437, 743

Kaufer, A., \& Pasquini, L. 1998, SPIE, 3355, 844

Kürster, M., Schmitt, J. H. M. M., Cutispoto, G., et al. 1997, A\&A, 320,831

Lowrance, P. J., Schneider, G., Kirkpatrick, J. D., et al. 2000, ApJ, 541,390

Pasquini, L., de Medeiros, J. R., \& Girardi, L. 2000, A\&A, 361, 1011

Queloz, D., Henry W., Sivan, J. P., et al. 2001, A\&A, 379, 279

Sato, B., Hiroyashu, A., Eiji, K., et al. 2003, ApJ, 597, L157

Scargle, J. D. 1982, ApJ, 263, 835

Schmidt-Kaler, Th. 1982, in Landolt-Börnstein Numerical Data and Functional Relationships and Technology, Vol. 2, Schaifers/Vogt (Berlin: Springer-Verlag)

Setiawan, J., Pasquini, L., da Silva, L., et al. 2003, A\&A, 397, 1151

Setiawan, J., Pasquini, L., da Silva, L., et al. 2004, A\&A, 421, 241

Setiawan, J., von der Lühe, O., da Silva, L., et al. 2005, in the Proceedings of the 13th Cool Stars Workshop, Cool Stars, Stellar Systems and the Sun 13, Hamburg, Germany 5-9 July 2004, ed. F. Favata et al., in press 